Formin' a perinuclear actin cage in confined migration

mechanically induced damage may be deregulated in cancer to enhance metastasis. AIZ

The mechanical stress exerted on a cell that translocates through confined 3D microenvironments can damage the nucleus and DNA. Waterman and colleagues report that FMN2, a member of the formin family of actin nucleators, mediates the formation of a perinuclear actin structure that protects the cell from nuclear damage during confined migration, thereby aiding cancer metastasis (Cell 167, 1571-1585; 2016).

The authors demonstrated that FMN2 was necessary for the formation of a system of perinuclear actin and focal adhesions at the dorsal surface of cultured cells, which supported nuclear positioning during 2D migration. A similar FMN2-actin network was observed in $3 \mathrm{D}$ collagen cell cultures. FMN2 depletion reduced the survival of cells migrating in this $3 \mathrm{D}$ system, which was associated with nuclear envelope rupture and DNA doublestrand breaks. This nucleus-protective role of FMN2 required its actin-nucleating activity. Exploring the role of FMN2 in a cancer setting, the authors showed that FMN2 mediated the formation of a perinuclear actin network in melanoma cells, and supported their survival during confined invasion in vitro. In vivo, FNM2 deficiency decreased melanoma metastasis, and increased the number of cells with DNA damage, whereas its overexpression had the opposite effect.

Thus, the FMN2-generated perinuclear actin cage that protects the nucleus from

\section{Force sensing in cytokinesis}

When an epithelial cell divides, the constriction of the cytokinetic ring is associated with a local accumulation of myosin II in the neighbouring cells and detachment at E-cadherin contacts at the site of the ring. Such junction remodelling and myosin II reorganization is essential for morphogenesis, but how the events above are coordinated is unclear.

Bellaïche and colleagues now find that reduced E-cadherin concentration, caused by forces generated from contractile ring constriction, results in actomyosin flow to concentrate myosin II in neighbouring cells (Nature 545, 103-107; 2017).

Through imaging and laser ablation experiments in Drosophila notum epithelium, the authors initially demonstrate that cytokinesis generates pulling forces, which induces myosin II accumulation in neighbouring cells. They also note that the local decrease in E-cadherin is accompanied by junction elongation and occurs prior to detachment from the neighbours and myosin II accumulation. Simulations and analysis of E-cadherin dynamics suggest that elongation of the junction results in dilution of E-cadherin, and indeed, lowering E-cadherin levels can rescue myosin II accumulation in neighbours in mutants with perturbed constriction. Further modelling shows that a decrease in friction

\section{EGFR probes matrix stiffness}

When cells migrate and spread, they mechanically test the rigidity of the extracellular matrix (ECM) by contracting it, and at certain levels of force, cells reinforce integrinmediated adhesions. Sheetz and colleagues now show that rigidity sensing is dependent on epidermal growth factor receptor (EGFR) activity, specifically on rigid surfaces (Nat. Mater. http://doi.org/b6wz; 2017).

Seeding cells onto micropillars coated with fibronectin confirms the role of local contractile units in contraction testing, and show that contractile unit activity is reduced on stiff compared with soft pillar matrix. EGFR is known to act with integrins in regulating cell-ECM interactions, and the authors find that loss of EGFR, through inhibition or knockdown, decreases spreading and contractility on stiff, but not on soft substrates. Interestingly, in the absence of EGFR, overexpression of the ligand-independent receptor HER2 can rescue rigidity sensing. The authors go on to show that EGFR can be activated in a ligand-independent manner by Src and, using inhibitors and mutants of Src-dependent phosphorylation sites, they find that this is needed for mechanosensing. However, EGF also stimulates local contraction events on rigid surfaces. Finally, they demonstrate that EGF-mediated activation of motility is dependent on myosin contractility and Src kinases.

These data demonstrate a previously unknown role for EGFR in rigidity sensing. CKR along the junction is sufficient to drive actomyosin flows in neighbouring cells, and timelapse experiments reveal reduced actomyosin flow and a delay in myosin II accumulation in mutants with less cytokinetic contractility and E-cadherin decrease.

These findings demonstrate the importance of actomyosin flow in force sensing and transmission during cytokinesis.

CKR

\section{Mechanical control of antigen uptake}

B-cells need to internalize antigen from antigen-presenting cells (APCs) to enable the expansion of high-affinity antibody-producing B-cell clones. Two mechanisms have been suggested for antigen extraction: enzymatic degradation of the antigen or a physical pull on it by the B-cell. Spillane and Tolar develop tools revealing that mechanical extraction dominates and is dependent on the physical properties of the APC membrane (J. Cell Biol. 216, 217-230; 2017)

The authors design a DNA-based fluorescent sensor that reports on mechanical versus enzymatic antigen extraction. Tethering antigen to substrates with mobility and stiffness shows that the type of extraction depends on the physical properties of the substrate. Combining this sensor with one that releases the antigen at a certain level of force applied by the B-cell demonstrates that mechanical extraction dominates. Further, stiff substrates promote selection of higher-affinity antigen, whereas flexible substrates mediate more efficient antigen extraction. They turn to experiments in which the sensor is loaded onto APCs relevant in vivo - follicular dendritic cells (FDCs) and dendritic cells (DCs). Atomic force microscopy shows that DCs have a more flexible plasma membrane than FDCs, and indeed DCs allow antigen extraction at weak forces, whereas FDCs promote better affinity discrimination, the latter dependent on actin.

These elegant experiments highlight the role of physical forces in antibody internalization. How forces are sensed by B-cells remains to be investigated. $\quad$ CKR

Written by Christina Karlsson Rosenthal and Alexia-lleana Zaromytidou 


\section{Retraction Note: Formin' a perinuclear actin cage in confined migration}

Alexia-lleana Zaromytidou

Retraction of: Nature Cell Biology https://doi.org/10.1038/ncb3547, published online 31 May 2017.

Given that the authors of 'FMN2 makes perinuclear actin to protect nuclei during confined migration and promote metastasis' (Skau et al. Cell 167, 1571-1585; 2016) have retracted their paper, I wish to retract this Research Highlight, which discussed the findings reported in that study. 